\title{
Acoustic Attenuation in Fans and Ducts by Vaporization of Liquid Droplets
}

\author{
Frank E. Marble* and Sebastien M. Candel ${ }^{\dagger}$ \\ California Institute of Technology, Pasadena, Calif.
}

\begin{abstract}
A cloud of small water droplets in saturated air attenuates acoustic disturbances by viscous drag, heat transfer, and vapor exchange with the ambient gas. The viscous and heat transfer phenomena attenuate at frequencies above $10^{4} \mathrm{~Hz}$ for $1-\mu$ droplets. The processes associated with phase exchange attenuate at a much lower frequency that may be controlled by choice of the liquid mass fraction. The strength of this attenuation is proportional to the mass of water vapor in the air, a factor controlled by air temperature. For plane waves, the attenuation magnitude exceeds $5 \mathrm{db} / \mathrm{m}$ at a temperature of $25^{\circ} \mathrm{C}$ with a cloud of $0.7 \mu$ radius droplets constituting $1 \%$ of the gas mass. This attenuation increases to more than $7 \mathrm{db} / \mathrm{m}$ at frequencies above $1000 \mathrm{~Hz}$ where viscous and heat transfer mechanisms contribute significantly. The attenuation of higher order duct modes is strongly increased over the above values, similarly to the attenuation by duct lining. When the droplet cloud occupies only a fraction of the duct height close to the walls, the droplet cloud may be up to twice as effective as the uniform cloud, and a significant saving is possible in the water required to saturate the air and furnish the water droplets.
\end{abstract}

\section{Introduction}

T HIS paper examines the extent that clouds of small liquid droplets may be employed to attenuate the acoustic strength of pure tones and duct modes and, in the process, to describe the physics and the analysis sufficiently to suggest possible extensions and technological applications. The acoustic attenuation properties of solid particles - more accurately, particles which have no mass transfer with gas supporting them - were examined in detail by Epstein ${ }^{1}$ and Epstein and Carhart. ${ }^{2}$ In recent years, the importance of particles or droplets of aluminum oxide in stabilizing high-frequency rocket instability has promoted considerable interest in the details of this process and in its nonlinear acoustics aspects. But for attenuation in linear acoustics, which is sufficient for the present investigation, the relevant quantities are the particle loading - ratio of solid to gas masses per unit volume - and the particle relaxation time or its reciprocal, the characteristic frequency.

Because the particle spacing-frequently less than $0.1 \mathrm{~mm}$ is small in comparison with the acoustic wave length, the action of the particulate phase may be represented as continuous distributions of weak acoustic sources and dipoles. In fact, it is immediately clear that the Stokes drag of the particles determines the dipole field and the heat exchange between gas and solid phase determines the thermal source field.

Investigations into the dynamics of multiphase mixture, Marble, ${ }^{3}$ led to the examination of the acoustics of droplet clouds, Wooten, ${ }^{4}$ Marble, and Wooten, ${ }^{5}$ in which vaporization and condensation of the droplet liquid accompanies the passage of an acoustic wave through the composite medium. The acoustic consequence of this phase transition appears as an additional effective source distribution, one portion of which is an actual mass source while the other is a thermal source. More striking physically, and certainly of more technological interest, is the natural division of those source fields associated with phase change in to components having short and long relaxation times.

Presented as Paper 74-526 at the AIAA 7th Fluid and Plasma Dynamics Conference, Palo Alto, California, June 17-19, 1974; submitted June 24, 1974; revision received November 5, 1974. Supported by U.S. Air Force Grant AFOSR-71 2068.

Index categories: Aircraft Noise, Aerodynamics (Including Sonic

Boom); Aircraft Noise, Powerplant; Multiphase Flows.

* Professor. Fellow AIAA

$\dagger$ Research Fellow; now Research Scientist, ONERA, Paris.
These two time scales relate physically to the distinct steps by which a vaporizing liquid attains equilibrium with its surroundings. ${ }^{5}$ The unique features of this low-frequency attenuation were beautifully confirmed in experiments of Cole and Dobbins. ${ }^{6}$

After the gas has undergone a weak compression and a correspondingly weak temperature rise, the droplet first absorbs heat from the gas, in much the same way and with the same relaxation time as if the droplet were inert. Before the droplet rises to equilibrium temperature, vaporization, in an effort to restore the vapor partial pressure to a thermodynamic equilibrium with the new droplet temperature, utilizes the influx of heat, and halts the rise in droplet temperature. Through the remainder of the equilibration process, the droplet temperature changes very slowly and the considerable heat flux from the warm gas is principally absorbed in supplying the latent heat of vaporization. Equilibration is achieved by adjusting the state of the entire gas rather than simply modifying the droplet temperature. The relaxation time for this process involves the mass of gas associated with each particle rather than the particle mass. Now when the particle loading is low, this relaxation is much larger, perhaps $10-10^{2}$ times, than the particle relaxation times appropriate to viscous and thermal attenuation. Therefore, significant acoustic absorption may be expected to take place at a much lower frequency and, in fact, one has reasonable technological control over this frequency through choice of particle loading.

The total amount of attenuation is proportional to the magnitude of heat or vapor exchange that occurs in this slow relaxation process. But to change the gas to its new equilibrium state, an amount of liquid must be vaporized to make the appropriate change in the vapor partial pressure. For a given strength of wave, this increment is proportional to the existing partial pressure of the vapor. Consequently, the strength of the attenuation is subject to control by choice of liquid volatility and the frequency may be selected by choice of liquid mass fraction.

In the following sections, the attenuation mechanism is examined in some detail, for low mass fractions of liquid, showing the relation between the various attenuation bands that constitute the complete process. With these quantitative results, it is shown how liquid properties, liquid mass fraction, and droplet size influence the attenuation. Then, in separate sections, this mechanism is applied to the attenuation of plane waves and duct modes associated with aircraft powerplants. 


\section{Weak Attenuation and Dispersion of Plane Waves}

Because the mass fraction and volume fraction of the liquid are very small, it is appropriate to consider the wave motion in the gaseous medium to be perturbed slightly by the small forces and mass and thermal sources arising from the second phase. Moreover, because the particle spacing is very small in comparison with acoustic wavelengths in the audible range, the effects of the liquid may be considered continuously distributed. As a consequence, they may be considered as an appropriately distributed set of weak acoustic sources and dipoles interacting with the wave train in question.

When the crests of a plane wave train are aligned normal to the $x$-axis, then the perturbation velocity $u$, pressure $p$, and density $\rho$ satisfies the linearized equations

$$
\begin{gathered}
\frac{\partial}{\partial t}\left(\frac{\rho}{\rho_{o}}\right)+c \frac{\partial}{\partial x}\left(\frac{u}{c}\right)=\frac{\phi}{\rho_{o}} \\
\frac{\partial}{\partial t}\left(\frac{u}{c}\right)+c \frac{\partial}{\partial x}\left(\frac{p}{\gamma p_{o}}\right)=\frac{F}{\rho_{o} c} \\
\frac{p}{\gamma p_{o}}-\frac{\rho}{\rho_{o}}=\frac{s}{c_{p}}
\end{gathered}
$$

where $\phi$ and $F$ are the weak mass sources and particle forces exerted on the gas, and $s$ is the entropy source of the gas resulting from heat and mass exchange with the liquid phase. The quantities $p_{o}, \rho_{o}$, and $c$ represent the undisturbed pressure, density, and acoustic velocity of the gaseous medium.

Then to treat weak attenuation and dispersion in this medium, consider a wave $P^{+} \exp i \omega(t-x / c)$ to impinge upon the medium with the result that the source and dipole fields modify this to

$$
\frac{p}{\gamma p_{o}}=P^{+} \exp i\left[\omega t-\left(\omega / c+k_{1}+i k_{2}\right) x\right]
$$

where $k_{1}$ and $k_{2}$ are the small real and imaginary parts of the wave number associated with the source and dipole fields. Now because the right-hand sides of Eqs. (1-3) are small, it is possible to write them to the correct approximation as

$$
\begin{aligned}
& \frac{\phi}{\rho_{o}}=\mathfrak{M}(\omega) P^{+} \exp i \omega(t-x / c) \\
& \frac{F}{\rho_{o}}=\mathfrak{F}(\omega) P^{+} \exp i \omega(t-x / c) \\
& \frac{s}{c_{p}}=\Im(\omega) P^{+} \exp i \omega(t-x / c)
\end{aligned}
$$

which states that they respond proportionally to the pressure (or velocity or temperature) of the impinging wave with amplitudes and phases that depend upon the frequency alone and are given by the three complex coefficients $\mathfrak{M}(\omega), \mathfrak{F}(\omega)$, and $\Theta(\omega)$. These three functions must be determined from an analysis of viscous drag, thermal exchange, and mass transfer between droplets and gas.

But before proceeding to this analysis, note that our acoustic equations in conventional form, eliminating the density fluctuation in Eq. (1) by using Eq. (3), become

$$
\begin{gathered}
\frac{\partial}{\partial t}\left(\frac{p}{\gamma p_{o}}\right)+c \frac{\partial}{\partial x}\left(\frac{u}{c}\right)=(\mathfrak{M}+i \omega \Im) P^{+} \exp i \omega(t-x / c) \\
\frac{\partial}{\partial t}\left(\frac{u}{c}\right)+c \frac{\partial}{\partial x}\left(\frac{p}{\gamma p_{o}}\right)=\frac{1}{c} \mathfrak{F} P^{+} \exp i \omega(t-x / c)
\end{gathered}
$$

so that the entropy term appears as a thermal source in the equation of continuity. As will appear later in the development, the thermal sources have the preponderant influence for the water-air system.

Now taking the solution in the proposed form Eq. (4) and retaining only linear terms in small quantities, the attenuation and dispersion coefficients are

$$
k_{1}+i k_{2}=(i / 2 c)(\mathfrak{M}+i \omega \Im+\mathfrak{F} / c) \equiv-(\omega / c) Q(\omega)
$$

and the desired attenuation coefficient follows directly from knowledge of the source-dipole field originating in the droplet cloud.

\section{Response of the Particle Cloud}

A droplet of mass $m$ and radius $a$, moving at a velocity $u_{p}$ in the gas velocity $u$ accompanying the wave motion, experiences a Stokes drag force $6 \pi a \mu\left(u-u_{p}\right)$ and consequently satisfies the equation of motion

$$
m \frac{\partial u_{p}}{\partial t}=6 \pi a \mu\left(u-u_{p}\right)
$$

Now because the particle cloud disturbs the gas motion very slightly, $u=c P^{+} \exp i \omega(x-t / c)$ and Eq. (11) determines the particle motion to be

$$
u_{p}=[1 /(1+i \omega \tau)] c P^{+} \exp i \omega(t-x / c)
$$

where $\tau$, the Stokes relaxation time, is $\tau=m / 6 \pi a \mu$. Then the total force $F$ exerted on the gas by the $n$ droplets per unit volume is

$$
F=(n m / \tau)\left(\frac{1}{1+i \omega \tau}-1\right) c P^{+} \exp i \omega(t-x / c)
$$

and from definition Eq. (6), the coefficient in the dipole strengths is simply

$$
\mathfrak{F}(\omega)=-\frac{\varkappa_{p} c}{\tau}\left(\frac{i \omega \tau}{1+i \omega \tau}\right)
$$

which determines directly the attenuation by viscous particle drag. Indeed, if this were the only effect, the attenuation coefficient $k_{2}$ would be, from Eq. (10),

$$
-(\omega / c)\left(x_{p} / 2\right)\left(\omega \tau / 1+\omega^{2} \tau^{2}\right)
$$

which is an attenuation band centered on the frequency $1 / \tau$, the reciprocal of the Stokes relaxation time.

The rate of vapor flow from the droplets into the gas phase, controlled by diffusion in the vapor phase, is driven by the difference between the vapor pressure at the droplet surface $p_{v}\left(T_{p}\right)$, where the vapor is in equilibrium with the liquid at the droplet temperature, and the partial pressure $p_{v}$ of the vapor in the bulk gas. The mass of vapor entering a unit volume occupied by $n$ droplets, in a medium with diffusion coefficient $D$, is then, for small deviations from equilibrium,

$$
\phi=n \cdot 4 \pi a(\rho D)\left[p_{v}\left(T_{p}\right)-p_{v}\right] / p_{o}
$$

Because the equilibrium vapor pressure at the droplet surface varies only slightly from the value $p_{v}\left(T_{o}\right) \equiv p_{v o}$ it would have if the entire system were in thermodynamic equilibrium, the difference is given by the Clapeyron relation

$$
p_{v}\left(T_{p}\right) / p_{v o}=\left(h_{l} / R_{v} T_{o}\right)\left(T_{p} / T_{o}\right)
$$

where now both $p_{v}\left(T_{p}\right)$ and $T_{p}$ represent perturbations from their respective equilibrium values and $R_{v}$ is the vapor gas constant. If we define a latent heat parameter

and the diffusion relaxation time

$$
\eta=h_{l} / c_{p} T_{o}
$$

$$
\tau_{D}=\frac{m}{4 \pi a(\rho D)}=\frac{3}{2} S c \cdot \tau
$$

$S c=\mu /(\rho D)$ being the Schmidt number for the gas mixture, the mass source strength is written

$$
\frac{\phi}{\rho_{o}}=\frac{\chi_{p} \varkappa_{v}}{\tau_{D}}\left(\beta \frac{\gamma}{\gamma-1} \eta \frac{T_{p}}{T_{o}}-\frac{p_{v}}{p_{v o}}\right)
$$

where $x_{v}$ is the ratio of vapor to total gas density, and $\beta$ is the molecular weight ratio of vapor to air.

The entropy source term, or perturbation of specific entropy, may be calculated most directly from the heat transfer rate to the (closed system) gas phase. The heat conducted from $n$ droplets of radius $a$ into a unit volume of gas having thermal conductivity $k$, is $n \cdot 4 \pi a k\left(T_{p}-T\right)$, which is, in turn, equal to the product of temperature and rate of entropy rise $T_{o}\left[\rho_{o}(\partial s / \partial t)\right]$. The process involves a thermal relaxation time

$$
\tau_{T}=m c_{p} / 4 \pi a k=(3 / 2) \operatorname{Pr} \cdot \tau
$$

and the differential equation for entropy source strength becomes

$$
\frac{\partial}{\partial t}\left(\frac{s}{c_{p}}\right)=\left(x_{p} / \tau_{T}\right)\left(T_{p}-T\right) / T_{o}
$$


Now to express the mass source Eq. (16) and the entropy source, Eq. (17), strengths in terms of the local unattenuated pressure fluctuation, three additional relationships-essentially thermodynamic - must be introduced. The first of these, the gas phase energy equations, is obtained by recalling the statement of the first law given by Eq. (3) and utilizing the entropy production rate aforementioned,

$$
\frac{\partial}{\partial t}\left(\frac{T}{T_{o}}\right)-(\gamma-1) \frac{\partial}{\partial t}\left(\frac{p}{\gamma p_{o}}\right)=\left(\varkappa_{p} / \tau_{T}\right)\left(T_{p}-T\right) / T_{o}
$$

The energy equation, or heat balance, for a droplet is somewhat more complicated because the droplet internal energy $m c_{l} T_{p}$ is reduced, not only by the heat transferred to the gas phase, but also the heat $h_{l}(\phi / n)$ which the droplet must supply to account for the evaporation from a single droplet. In terms of our variables and parameters,

$$
\chi_{p}\left(\frac{c_{l}}{c_{p}}\right) \frac{\partial}{\partial t}\left(\frac{T_{p}}{T_{o}}\right)=-\left(\frac{\chi_{p}}{\tau_{T}}\right) \frac{\left(T_{p}-T\right)}{T_{o}}-\eta\left(\frac{\phi}{\rho_{o}}\right)
$$

Finally, we require a relation describing how the vapor source strength affects the partial pressure of the vapor in the bulk gas. Beginning with continuity equation for the vapor density $\partial \rho_{v} / \partial t+\rho_{v o}(\partial u / \partial x)=\phi$ and using the bulk equation of continuity, Eq. (1), to eliminate the velocity gradient, it follows that

$$
\frac{\partial}{\partial t}\left(\frac{\rho_{v}}{\rho_{v o}}-\frac{\rho}{\rho_{o}}\right)=\left(\frac{1-\varkappa_{v}}{\chi_{v}}\right) \frac{\phi}{\rho_{o}}
$$

But because the temperature of the bulk gas and temperature of the vapor are identical, and we have assumed the gas properties to be indisguishable, the above equation becomes

$$
\frac{\partial}{\partial t}\left(\frac{p_{v}}{p_{v o}}-\frac{p}{p_{o}}\right)=\left(\frac{1-\chi_{v}}{\chi_{v}}\right) \frac{\phi}{\rho_{o}}
$$

the thermodynamic relation that was desired.

Equations (16) and (18-20), describe the response of the distributed mass and heat sources, originating in the droplets and their phase change, as they are acted upon by the unattenuated pressure wave. Each of the quantities $T / T_{o}, T_{p} / T_{o}, \phi / \rho_{o}$, and $p_{v} / p_{v o}$, is proportional to $P^{+} \exp i \omega(t-x / c)$, the quantity of proportionality being in each case a complex function of frequency and physical parameters. Entering this representation into each of the equations, a set of algebraic equations is obtained for the response functions, each of which follows by a conventional but tedious calculation. It is of special interest that the determinant of the coefficients, which forms the denominator of each response function, is simply the product of $i \omega$ and a second-degree polynomial in $i \omega$ with the consequence that the system distinguishes two frequencies.

$$
\begin{aligned}
& \Omega_{2}=\frac{1}{\tau_{T}} \frac{c_{p}}{c_{l}}\left[1+\operatorname{Lex}_{v} \beta \frac{\gamma}{\gamma-1} \eta^{2}\right] \\
& \Omega_{3}=\frac{x_{p}}{\tau_{D}}\left[\frac{1-x_{v}+x_{v} \beta(\gamma / \gamma-1) \eta^{2}}{1+\operatorname{Lex}_{v} \beta(\gamma / \gamma-1) \eta^{2}}\right]
\end{aligned}
$$

We reserve the notation

$$
\Omega_{1}=1 / \tau
$$

for the frequency associated with the Stokes relaxation time and characterizing the dipole field. The Lewis number, appearing in Eqs. (21) and (22), is the ratio of Prandtl number to Schmidt number, $L e \equiv \operatorname{Pr} / S c$.

The frequency $\Omega_{2}$ is very close to the Stokes relaxation frequency for small values of the latent heat parameter $\eta$ and increases to perhaps several times $\Omega_{1}$ for large values of $\eta$. The frequency $\Omega_{3}$, however, is of the order $\chi_{p} \Omega_{1}$, and hence much smaller than $\Omega_{1}$, the influence of latent heat being unimportant. Most significantly, from a technological viewpoint, $\Omega_{3}$ may be as low as one per cent of $\Omega_{1}$ and consequently lie in an interesting audio acoustic range. The physical origin of this very long relaxation time will be examined presently.

Now because the determinant may be factorized, the response functions for the mass source $\mathfrak{M}(\omega)$ and for their thermal or entropy source $\Xi(\omega)$ [Eqs. (5) and (7)] may be written, using standard techniques, as the sum of responses associated with each of the frequencies $\Omega_{2}$ and $\Omega_{3}$. In this representation, the mass source response $\mathfrak{M}(\omega)$ takes the form

$$
\begin{aligned}
-\frac{c_{l}}{c_{p}} \operatorname{Lex}_{p} x_{v} \frac{\left[1+\operatorname{Lex}_{v}(\gamma / \gamma-1) \eta\right] \beta \eta}{[1}+\operatorname{Lex_{v}\beta (\gamma /\gamma -1)\eta ^{2})^{2}} \frac{i \omega \gamma}{1+\left(i \omega / \Omega_{2}\right)} \\
\quad+x_{v}\left[\frac{\beta \eta-1}{1-\chi_{v}+x_{v} \beta(\gamma / \gamma-1) \eta^{2}}\right] \frac{i \omega \gamma}{1+\left(i \omega / \Omega_{3}\right)}
\end{aligned}
$$

and the entropy response $i \omega \subseteq(\omega)$ is similarly

$$
\begin{aligned}
& -\frac{\gamma-1}{\gamma} \frac{c_{t}}{c_{p}} x_{p}\left[1+\frac{1+\operatorname{Lex}_{v}(\gamma / \gamma-1) \eta}{\left.\operatorname{Lex} \beta(\gamma / \gamma-1) \eta^{2}\right]^{2}} \frac{i \omega \gamma}{1+i\left(\omega / \Omega_{2}\right)}\right. \\
& -x_{v} \frac{\eta(\beta \eta-1)}{1-\varkappa_{v}+\chi_{v} \beta(\gamma / \gamma-1) \eta^{2}} \frac{i \omega \gamma}{1+i\left(\omega / \Omega_{3}\right)}
\end{aligned}
$$

It is worth noting here that, in each case, the magnitude of high-frequency response term $\left(\omega / \Omega_{2}\right)$ is proportional to the liquid mass fraction $\chi_{p}$, while the magnitude of the low-frequency response $\left(\omega / \Omega_{3}\right)$ is independent of $x_{p}$ but proportional to the equilibrium vapor mass fraction $\chi_{v}$. The physical basis for this, which has been suggested in the Introduction, is clarified by examining the corresponding response function $\mathfrak{I}_{p}(\omega)$ in the expression $T_{p} / T_{o} \equiv \mathfrak{I}_{p}(\omega) P^{+} \exp i \omega(t-x / c)$, for the result

$$
\mathfrak{I}_{p}(\omega)=(\gamma-1)\left[\frac{1+\operatorname{Lex}_{v}(\gamma / \gamma-1) \eta}{1+\operatorname{Lex}_{v} \beta(\gamma / \gamma-1) \eta^{2}}\right] \frac{1}{1+i\left(\omega / \Omega_{2}\right)}
$$

has only a part corresponding to the high-frequency response and, to the first order of approximation, none corresponding to the slow relaxation process. Referring to droplet temperature, Eq. (19), this result indicates that a fluctuation in the heat transfer and latent heat absorption causes a brief adjustment in the droplet temperature (in a time $\sim 1 / \Omega_{2}$ ) and subsequently the droplet temperature remains nearly constant. This in turn demonstrates that for larger times the heat transferred to droplet is balanced by the latent heat of vaporization being carried away from the droplet. The droplets simply act as agents to absorb heat from the bulk gas and, in turn, supply vapor to the bulk gas until thermodynamic equilibrium of the mixture is achieved. The droplet temperature, after its initial adjustment to a value intermediate between its initial value and the bulk gas temperature, takes little further part in the equilibration process. This droplet temperature is simply the classical "wet bulb" temperature of the mixture.

These observations on Eqs. (23-25) confirm our earlier conjectures on the origin of the long relaxation time. The shorttime particle response is one in which the particle state is being changed; the mass being altered is simply $m$, the droplet mass; the relevant relaxation time is $m c_{p} / 4 \pi a k$. But after the droplet reaches its "wet bulb" temperature, the state of the bulk gas, having a mass $m / \chi_{p}$ associated with each particle, must be altered by similar exchange processes and hence the relevant relaxation time is $m c_{p} / x_{p} 4 \pi a k$.

The magnitudes of the thermal (entropy) and mass responses also can be understood physically. For high-frequency response, the state of the particulate mass is changing, and therefore the exchange and the attenuation are both proportional to that mass fraction, namely $\varkappa_{p}$. For lower frequencies, it is the state of the gas mass which is being altered, and its thermodynamic

Table 1 Variation of $\Omega_{2}$ and $\Omega_{3}$ with air temperature

\begin{tabular}{rcc}
\hline \hline${ }^{\circ} \mathrm{C}$ & $\Omega_{2} / \Omega_{1}$ & $\Omega_{3} / \varkappa_{p} \Omega_{1}$ \\
\hline 5 & 0.46 & 1.05 \\
10 & 0.55 & 1.03 \\
15 & 0.67 & 1.01 \\
20 & 0.83 & 1.00 \\
25 & 0.99 & 0.99 \\
30 & 1.19 & 0.98 \\
40 & 1.43 & 0.97 \\
50 & 2.16 & 0.96
\end{tabular}


equilibrium depends upon adjusting the partial pressure of vapor; the mass exchange and attenuation therefore are both proportional to mass fraction $x_{v}$ of vapor, a fact that is reflected in the second terms of Eqs. (23) and (24).

\section{Acoustic Absorption Strength-Attenuation of Plane Waves}

With the response of the source and dipole fields known, the attenuation wave number $k_{2}$ follows directly from Eq. (10) as $k_{2}=-(\omega / c) \mathscr{I}_{m}(Q)$. Utilizing the results of Eqs. (13, 23, and 24), the attenuation wave number may be written as

$$
\begin{aligned}
k_{2}=-\frac{1}{2} \frac{\omega}{c}\left\{\chi_{p} \frac{\omega / \Omega_{1}}{1+\left(\omega / \Omega_{1}\right)^{2}}+\right. & \\
& \left.x_{p} g_{2} \frac{\omega / \Omega_{2}}{1+\left(\omega / \Omega_{2}\right)^{2}}+g_{3} \frac{\omega / \Omega_{3}}{1+\left(\omega / \Omega_{3}\right)^{2}}\right\}
\end{aligned}
$$

where the functions $g_{2}$ and $g_{3}$ depend upon the thermodynamic parameters of the system and are

$$
\begin{gathered}
g_{2}=(\gamma-1) \frac{c_{l}}{c_{p}} \frac{\left[1+\operatorname{Lex}_{v}(\gamma / \gamma-1) \eta\right]\left[1+\operatorname{Lex}_{v} \beta(\gamma / \gamma-1) \eta\right]}{\left[1+\operatorname{Lex}_{v} \beta(\gamma / \gamma-1) \eta^{2}\right)^{2}} \\
g_{3}=\gamma \varkappa_{v} \frac{(\eta-1)(\beta \eta-1)}{1-\chi_{v}+x_{v} \beta(\gamma / \gamma-1) \eta^{2}}
\end{gathered}
$$

Each term within the brackets of Eq. (26) represents an "absorption band" in the frequency variable; each one has its maximum at its characteristic frequency, $\Omega_{1}, \Omega_{2}$, or $\Omega_{3}$. The absorption bands corresponding to $\Omega_{1}$ and $\Omega_{2}$ usually overlap. For systems of interest in the present investigation, that is, where $x_{p} \ll 1$, the low-frequency band centered on $\Omega_{3}$ is usually separate from the others and is the most susceptible to technological control. Both the frequencies and the strengths of attenuation at each frequency are dependent upon the thermodynamics of the liquid-vapor-gas system, and it is instructive to examine the example of the water-water vapor-air system that is of central interest here.

The frequency $\Omega_{1}$ is simply the inverse of the velocity relaxation time which, for an ambient pressure of one atmosphere and a temperature of $15^{\circ} \mathrm{C}$, gives a value of $\Omega_{1}=$ $0.806 \times 10^{5} \mathrm{rad} / \mathrm{sec}$ for a droplet radius of $1 \mu$. This frequency varies inversely as the square of the droplet radius and directly as the air viscosity, so that, in general,

$$
\Omega_{1}=0.806 \times 10^{5}(a)^{-2}\left(\mu / \mu_{o}\right)
$$

where $a$ is now the droplet radius in microns and $\mu_{0}$ is the viscosity of air at $15^{\circ} \mathrm{C}$. The frequencies $\Omega_{2}$ and $\Omega_{3}$ scale correspondingly with droplet radius so that their temperature dependence, which enters strongly through the saturation vapor pressure or $x_{v}$, may be separated. Therefore, it is convenient to tabulate the quantities $\Omega_{2} / \Omega_{1}$ and $\Omega_{3} / x_{p} \Omega_{1}$ because the variation of each ratio from unity depends only upon the physical properties of the mixture and the mixture temperature. For example, then, the value of $\Omega_{3}$ for a droplet cloud, in saturated air at $30^{\circ} \mathrm{C}$, consisting of $2 \%$ by weight of droplets having $0.5 \mu$ radius, is $\Omega_{3}=668 / \mathrm{sec}$, to be compared with $\Omega_{1}=3.39 \times 10^{5} / \mathrm{sec}$ and $\Omega_{2}=4.07 \times 10^{5} / \mathrm{sec}$ for the same conditions.

The magnitudes of attenuation require evaluation of the functions $g_{2}$ and $g_{3}$, which depend upon only the thermo-

Table 2 Attenuation strength functions, $g_{2}$ and $g_{3}$

\begin{tabular}{rcc}
\hline \hline$T^{\circ} \mathrm{C}$ & $g_{2}$ & $g_{3}$ \\
\hline 5 & 0.54 & 0.13 \\
10 & 0.43 & 0.15 \\
15 & 0.33 & 0.18 \\
20 & 0.25 & 0.20 \\
25 & 0.20 & 0.21 \\
30 & 0.17 & 0.22 \\
40 & 0.14 & 0.23 \\
50 & 0.11 & 0.24 \\
\hline
\end{tabular}

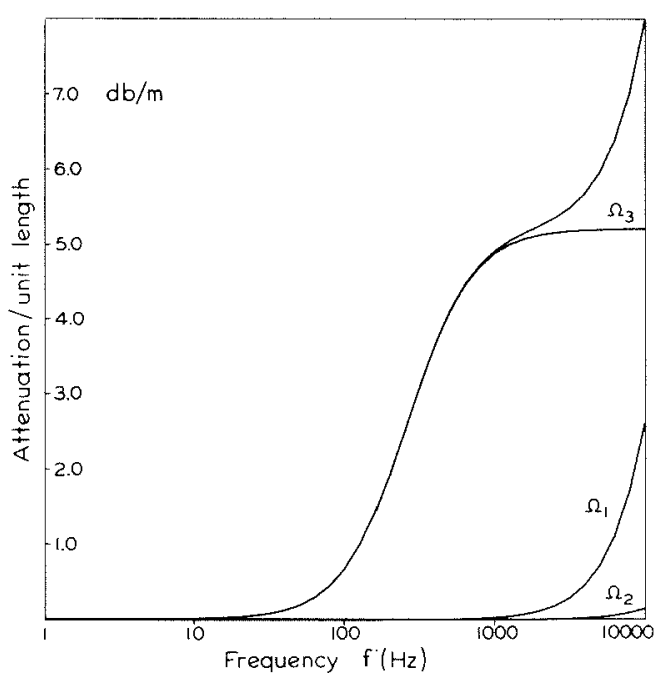

Fig. 1 Attenuation of plane acoustic waves by water droplets, $x_{p}=$ $0.010, a=0.7 \mu, T_{o}=25^{\circ} \mathrm{C}$.

dynamic variables. For an ambient pressure of one atmosphere, these quantities are given in Table 2 as they vary over a reasonable range of temperature. It is particularly noted that these values are independent of droplet size and the mass fraction of liquid present.

The actual attenuation of plane waves may now be evaluated using Eq. (26) in conjunction with the material from Tables 1 and 2. For applications to powerplants and ducts, it is convenient to give attenuation values in decibels per meter of wave travel, and this has been shown in Fig. 1 for a specific example of a droplet mass fraction $\chi_{p}=0.01$ with droplet radius of $0.7 \mu$ in atmosphere air at $25^{\circ} \mathrm{C}$. The separate contributions of momentum exchange $\left(\Omega_{1}\right)$, heat transfer $\left(\Omega_{2}\right)$, and phase equilibration $\left(\Omega_{3}\right)$ are indicated by their characteristic frequencies. The total attenuation appears as the unmarked curve. Note particularly that the attenuation associated with $\Omega_{3}$ appears at the lowest impressed frequency and attains a value of nearly $5 \mathrm{db} / \mathrm{m}$ before the viscous drag effects $\left(\Omega_{1}\right)$ begin to be significant at about $10^{3} \mathrm{~Hz}$

Note also from Eq. (26) that when discussing attenuation per unit length, the attenuation "bands" described earlier become "steps" because of the factor $\omega$ appearing in the attenuation wave number. For each contribution the rise occurs around the appropriate frequency and attains a value proportional to that

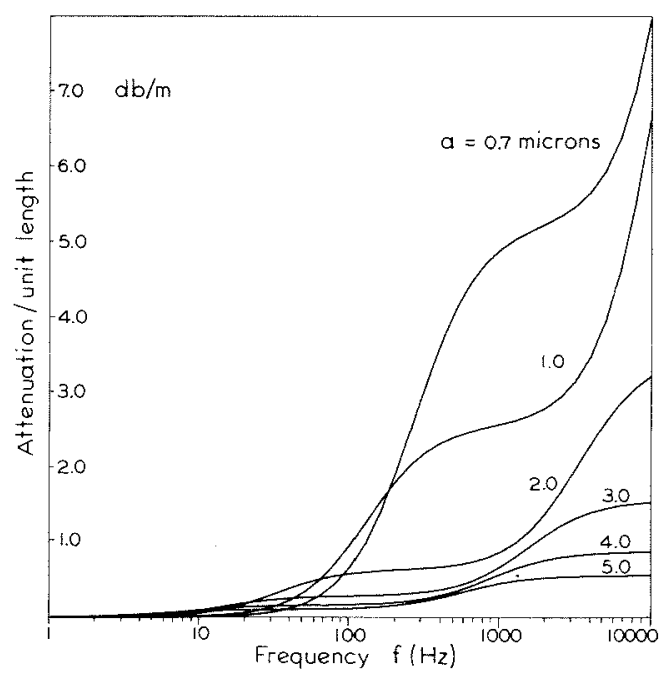

Fig. 2 Attenuation of plane acoustic waves by water droplets of various radii, $\varkappa_{D}=0.01, T_{0}=25^{\circ} \mathrm{C}$ 
characteristic frequency. Therefore, if all parameters are fixed (including $x_{p}$ ) and the droplet radius is increased, each characteristic frequency decreases as the square of the droplet radius and reaches a correspondingly smaller plateau value with impressed frequency. This sensitivity to droplet radius is demonstrated in Fig. 2. The importance of obtaining small droplet radii is clear; if the droplet radius were allowed to increase to $2 \mu$ instead of the $0.7 \mu$ radius in the original example, the attenuation at $10^{3} \mathrm{~Hz}$ would be reduced by a factor of about 5 , and indeed, the contribution of phase change is not particularly significant.

If the conditions of Fig. 1 are applied to the inlet of a current turbofan engine handling $600 \mathrm{~kg} / \mathrm{sec}$ of air at takeoff, it is found that for an ambient relative humidity of $50 \%$, the water injection requirement is about $8 \mathrm{~kg} / \mathrm{sec}$ during the critical part of a landing or takeoff maneuver. It will appear in the next section that this requirement may be relieved by altering the spatial distribution of droplets.

\section{Attenuation of Duct Modes}

Propagating pressure modes in a two-dimensional hard-walled duct of height $l$, have the form $p / \gamma p_{o}=P^{+} \exp i \omega(t-x \cos v / c)$. $\cos (n \pi y / l)$, constructed from plane wave trains the normals to which make angles

$$
\pm v=\cos ^{-1}\left\{1-(n \pi c / \omega l)^{2}\right\}^{1 / 2}
$$

with the axis of the duct. When the duct is uniformly filled with a disperse cloud of liquid droplets, it may be expected that, because the transverse wave number $k_{y}=n \pi / l$ is fixed by the wall boundary conditions, the attenuation and dispersion will appear as modifications in the $x$-component of wave number to

$$
k_{x}=(\omega / c) \cos v+k_{x 1}+i k_{x 2}
$$

the perturbation $k_{x_{1}}+i k_{x_{2}}$ being of the same small order as the distributed source and dipole strength of the droplet cloud. The relevant wave equation is developed in much the same manner as the one-dimensional equation except that both $x$ - and $y$ components of the particle force must be accounted for, with the result that

$$
\begin{aligned}
\left\{\frac{\partial^{2}}{\partial t^{2}}-c^{2}\left(\frac{\partial^{2}}{\partial x^{2}}+\frac{\partial^{2}}{\partial y^{2}}\right)\right\} \frac{p}{\gamma p_{o}} & = \\
-\frac{1}{2} \omega Q(\omega) P^{v} \exp & {\left[i \omega\left(t-\frac{x \cos v}{c}\right)\right] \cos \frac{n \pi y}{l} }
\end{aligned}
$$

the notation being identical with that used earlier. The pressure will be altered only slightly from the unattenuated modes, through the perturbed wave number, and in fact this perturbation is just

$$
k_{x 1}+i k_{x 2}=-(\omega / c)(1 / \cos v) Q(\omega)
$$

This result is identical with that for plane waves, Eq. (10), except for the factor $1 / \cos v$ multiplying the attenuation coefficient for duct modes. The new factor is expected intuitively from geometrical acoustics, because the mode is constructed of plane waves with wave crests inclined at $\pm v$ with the vertical and the distance traveled by the waves is simply $1 / \cos v$ times the distance traveled along the duct axis.

In practical applications of droplet attenuation it is most likely that the droplet cloud will be confined to a region close to the duct walls, where injection of the droplets is more easily achieved. To explore this situation, consider the duct of height $l$ to be filled with a droplet cloud to a depth $d=\alpha l$ extending from the lower wall. The solution must be represented separately in each region, and if these representations are chosen to satisfy identically the conditions that the normal velocity vanishes at both walls,

$$
\begin{aligned}
& \frac{p}{\gamma p_{o}}=P^{v} \exp i\left(\omega t-k_{x} x\right) \cos k_{y}(l-y) \quad \alpha l<y \leqq l \\
& \frac{p}{\gamma p_{o}}=P^{\prime v} \exp i\left(\omega t-k_{x} x\right) \cos k_{y}^{\prime} y \quad 0 \leqq y<\alpha l
\end{aligned}
$$

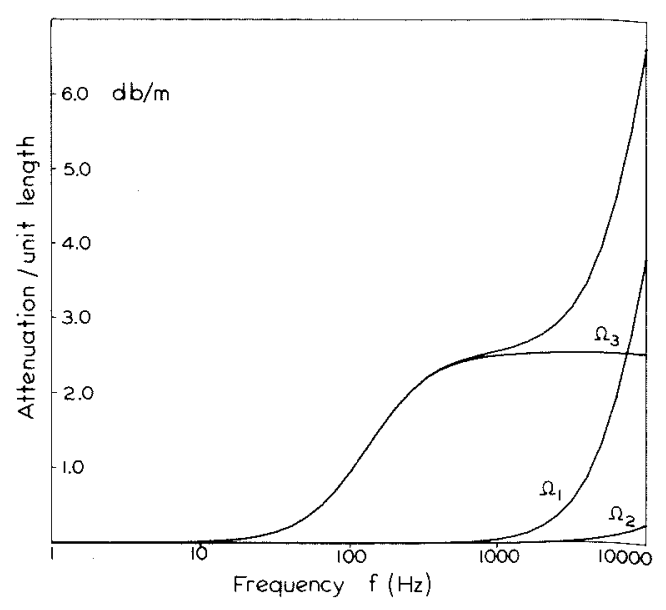

Fig. 3 Attenuation of plane wave in duct containing uniform droplet cloud, $x_{p}=0.01, a=1 \mu, T_{o}=25^{\circ} \mathrm{C}$.

To satisfy the conditions that both the perturbation pressure and the vertical velocity match at the interface $y=\alpha l$, it is required that the $x$-wave number be the same for both solutions, and thus the $y$-wave numbers may differ only because of the inhomogeneous Eq. (31) to be satisfied in the lower region. Because the solution is only slightly perturbed from unattenuated modes, the $y$-wave numbers will be only slightly perturbed from $n \pi / l$ and hence it is appropriate to choose

$$
\begin{aligned}
& k_{y}=(n \pi / l)+k_{y 1} \\
& k_{y}^{\prime}=(n \pi / l)+k_{y 1}^{\prime}
\end{aligned}
$$

But for the pressure fields Eq. (33) to be solutions of their respective wave equations, it is required that to the first order, the difference between the two $y$-wave numbers is

$$
k_{y 1}^{\prime}-k_{y 1}=-(\omega / c)(\omega l / n \pi c) Q(\omega)
$$

Now in the absence of mean motion, the two solutions are matched at $y=\alpha l$ by making $p / \gamma p_{o}$ and $\partial / \partial y\left(p / \gamma p_{o}\right)$ continuous. This process completes the characteristic condition on the $y$-wave number and by standard techniques, utilizing Eqs. (34) and (35), the wave number perturbation is found to be

$$
k_{y 1}=\left(1+\frac{\sin 2 \alpha n \pi}{2 \alpha n \pi}\right) \propto\left(\frac{\omega}{c}\right)\left(\frac{\omega l}{n \pi c}\right) Q(\omega)
$$

from which the $x$-wave number required to assure that expressions (33) are solutions to the wave equation is

$k_{x}=\left(\frac{\omega}{c}\right) \cos v-\left(\frac{\omega}{c}\right)\left(\frac{1}{\cos v}\right)\left(1+\frac{\sin 2 \alpha n \pi}{2 a n \pi}\right) \alpha Q(\omega)$

The attenuation coefficient is thus simply related to that for the duct uniformly covered with a droplet cloud; the attenuation for the duct with droplet layer covering a fraction $\alpha$ of the width adjacent to one wall is

$$
\left(1+\frac{\sin 2 \alpha n \pi}{2 \alpha n \pi}\right) \alpha
$$

times that for the uniform cloud. An exception must be made for the longitudinal wave in the duct, the mode $n=0$, for which the perturbation to the $x$-wave number is simply $(\omega / c) \alpha Q(\omega)$ instead of twice that value which would be indicated by a limiting process on Eq. (37). That the case $n=0$ must be considered separately is physically apparent because the orientation of the wave crest normal excludes a fraction $1-\alpha$ of the wave from the particle cloud and this part is weakened by a mechanism quite different from that for higher modes.

In considering the practical advantages of utilizing layers of droplets close to the walls, two factors must be taken into account. The first is simply the magnitude of the attenuation for a given droplet and vapor density, and this is provided by Eq. (37) [or (36)]. The second is the actual mass of liquid that is being employed, and this is generally a fraction $\alpha$ of that required for uniform droplet coverage. The effectiveness of liquid 


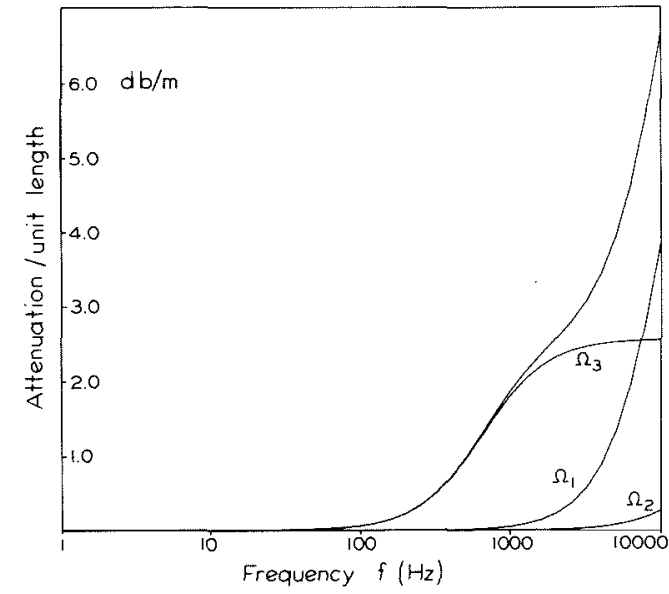

Fig. 4 Attenuation of plane wave in duct, one wall covered by water droplet cloud to depth of 0.2 duct widths; $\varkappa_{p}=0.05, a=1 \mu, T_{o}=25^{\circ} \mathrm{C}$

usage is therefore measured by $1+(\sin 2 \alpha n \pi / 2 \alpha n \pi)$ and this varies, for the first duct mode, from the value 2 for very thin droplet layers to a value of 1 for half coverage. In general, then, the droplets may be utilized more effectively near the duct wall.

If we ask for the best location for a fixed quantity of liquid droplets, that is, shall it be uniformly distributed or concentrated near the walls, the dependence of $Q(\omega)$ upon the particle concentration must be considered. Although the low frequency component $\left(\Omega_{3}\right)$ is independent of $\chi_{p}$, the viscous $\left(\Omega_{1}\right)$ and heat transfer $\left(\Omega_{2}\right)$ components increase linearly with $\chi_{p}$ and thus the product $\alpha \varkappa_{p}$ remains constant as the same quantity of droplets is concentrated in a layer adjacent to the wall.

An example will clarify the effect of layered clouds. Consider the attenuation of the normal wave in a duct. Suppose the duct to be uniformly filled with a cloud of $1-\mu$ radius droplets with mass fraction $\chi_{p}=0.01$ while the mixture is at a temperature of $25^{\circ} \mathrm{C}$. The attenuation, the components and total of which are given in Fig. 3, show the low-frequency $\left(\Omega_{3}\right)$ attenuation appearing significantly at $100 \mathrm{~Hz}$, reaching its plateau of about $2.5 \mathrm{db} / \mathrm{m}$ at $1000 \mathrm{~Hz}$. The high-frequency viscous and heat transfer attenuations become significant above $2000 \mathrm{~Hz}$.

Now let the cloud be confined to the region between the wall and a distance $0.2 l$ above the wall, the stream temperature and droplet radius being the same as for the uniform cloud, but the total droplet mass chosen identical in both cases so that within the layer, $x_{p}=0.05$ and vanishes outside the layer. Now because $\alpha x_{p}$ is the same in both cases, the high-frequency $\left(\Omega_{1}, \Omega_{2}\right)$ attenuation is unaffected by redistributing the droplets. The low-frequency attenuation is more complicated because the increase in $\chi_{p}$ [cf. Eq. (22)] increases the frequency $\Omega_{3}$ in proportion, and hence this attenuation mechanism appears at frequencies five times the value for uniform coverage. Reference to Eq. (26) shows that for any value of $\omega / \Omega_{3}$, the low-frequency attenuation is proportional to $\Omega_{3}$. But for the layered cloud $\alpha \varkappa_{p} \sim \alpha \Omega_{3}$ is the same as for the uniform cloud. The low-frequency attenuation for the layered cloud will have the same value as for the uniform cloud, but will appear at a frequency 5 times as high. The contributions of each attenuation mechanism are given in Fig. 4, and these detailed calculations confirm the previous discussion.

It should be noted that in spite of the fact that total droplet mass is the same for both uniform and layered clouds, there is a considerable saving in water flow rate because only $20 \%$ of the air flowing must be saturated with water vapor. Supposing that the initial relative humidity is $50 \%$, and knowing that within the particle cloud at $25^{\circ} \mathrm{C}$ the value $\chi_{v}$ is 0.019 , the total water requirement is only $60 \%$ of that for the uniform cloud and identical attenuation is achieved for frequencies above $1500 \mathrm{~Hz}$.

Finally, the relationship between droplet attenuation and acoustical duct lining will be indicated because both produce similar effects by quite different mechanisms. Now a duct lining or soft boundary may be expressed as a specific acoustical admittance which, when applied to the lower wall of the duct replaces the condition $v(x, 0, t)=0$ with

$$
\left(-\frac{v}{c}\right) / \frac{p}{\gamma p_{o}}=\xi-i \sigma
$$

at $y=0$, the complex admittance $\xi-i \sigma$ being fixed by the known properties of the liner. By quite standard perturbation methods the $x$-wave number, in the presence of one lined surface, is found to be

$$
\begin{aligned}
k_{x} & =(\omega / c) \cos v-(1 / \cos v)(1 / l)(\sigma+i \xi) \quad n=1,2, \ldots \\
& =\omega / c-\frac{1}{2}(\sigma+i \xi) \quad n=0
\end{aligned}
$$

By comparison with the results of the droplet layer analysis, Eq. (37), the droplet attenuation corresponds to a complex surface admittance

$$
\xi-i \sigma=-\left(\frac{i \omega l}{c}\right)\left(1+\frac{\sin 2 \alpha n \pi}{2 \alpha n \pi}\right) \alpha Q
$$

and hence, the two techniques of attenuation may be compared directly.

\section{References}

${ }^{1}$ Epstein, P. S., Contributions to Applied Mechanics, T. von Kármán Anniversary Volume, Calif. Institute of Tech., Pasadena, Calif., 1941, pp. $162-188$.

${ }^{2}$ Epstein, P. S. and Carhart, R. R., Journal of the Acoustical Society of America, Vol. 25, 1955, pp. 555-565.

${ }^{3}$ Marble, F. E., "Some Gasdynamic Problems in the Flow of Condensing Vapors," Astronautica Acta, Vol. 14, Oct. 1969, pp. 585-614.

${ }^{4}$ Wooten, D. C., "The Attenuation and Dispersion of Sound in a Condensing Medium," Pt. I, Ph.D. thesis, California Institute of Technology, 1966, pp. 1-72.

${ }^{5}$ Marble, F. E. and Wooten, D. C., "Sound Attenuation in a Condensing Vapor," Physics of Fluids, Vol. 13, Nov. 1970, pp. 26572664.

${ }^{6}$ Cole, J. E. III and Dobbins, R. A.. Journal of the Atmospheric Sciences, "Measurements of the Attenuation of Sound by a Warm Air Fog," Vol. 28, March 1971, pp. 202-209. 